\title{
DIEUDONNÉ-SCHWARTZ THEOREM IN INDUCTIVE LIMITS OF METRIZABLE SPACES II
}

\author{
QIU JING HUI
}

(Communicated by William J. Davis)

\begin{abstract}
The Dieudonné-Schwartz Theorem for bounded sets in strict inductive limits does not hold for general inductive limits $E=$ ind $\lim E_{n}$. It does if all the $E_{n}$ are Fréchet spaces and for any $n \in N$ there is $m(n) \in N$ such that $\bar{E}_{n}^{E_{p}} \subset E_{m(n)}$ for all $p \geq m(n)$. A counterexample shows that this condition is not necessary. When $E$ is a strict inductive limit of metrizable spaces $E_{n}$, this condition is equivalent to the condition that each bounded set in $E$ is contained and bounded in some $\left(E_{n}, \xi_{n}\right)$. Also, some interesting results for bounded sets in inductive limits of Fréchet spaces are given.
\end{abstract}

Let $E_{1} \subset E_{2} \subset \cdots$ be a sequence of locally convex spaces and $(E, \xi)=$ ind $\lim \left(E_{n}, \xi_{n}\right)$ their inductive limit with respect to the continuous identity maps id: $\left(E_{n}, \xi_{n}\right) \rightarrow\left(E_{n+1}, \xi_{n+1}\right)$. The Dieudonné-Schwartz Theorem [1, Chapter $2, \S 12]$ states that a set $B \subset E$ is $\xi$-bounded if and only if it is contained and bounded in some $\left(E_{n}, \xi_{n}\right)$, provided that $(E, \xi)=\operatorname{ind} \lim \left(E_{n}, \xi_{n}\right)$ is a strict inductive limit and each $E_{n}$ is closed in $\left(E_{n+1}, \xi_{n+1}\right)$. In [2]-[5], it has been extended to inductive limits. For brevity, denote by

(DS) each set $B$ bounded in $(E, \xi)$ is contained in some $E_{n}$;

(DST) each set $B$ bounded in $(E, \xi)$ is contained and bounded in some $\left(E_{n}, \xi_{n}\right)$.

1. It was proved in [5] that if all $\left(E_{n}, \xi_{n}\right)$ are Fréchet spaces and for any $n \in N$ there is $m(n) \in N$ such that $\bar{E}_{n}^{E} \subset E_{m(n)}$ then (DST) holds, where $\bar{E}_{n}^{E}$ is the closure of $E_{n}$ in $(E, \xi)$; see [5, Theorem 1]. Since the closure of $E_{n}$ in $(E, \xi)$ may be difficult to construct, the assumption $\bar{E}_{n}^{E} \subset E_{m(n)}$ is not practical. This situation is remedied in the following.

Theorem 1. Let all $\left(E_{n}, \xi_{n}\right)$ be Fréchet spaces. Then (DST) holds provided that for any $n \in N$ there is $m(n) \in N$ such that $\bar{E}_{n}^{E_{p}} \subset E_{m(n)}$ for all $p \geq m(n)$, where $\bar{E}_{n}^{E_{p}}$ is the closure of $E_{n}$ in $\left(E_{p}, \xi_{p}\right)$.

Received by the editors June 15, 1988 and, in revised form, April 30, 1989.

1980 Mathematics Subject Classification (1985 Revision). Primary 46A05.

Key words and phrases. Locally convex spaces, (strict) inductive limit, bounded set. 
Proof. Assume that $\bar{E}_{n}^{E_{p}} \subset E_{m(n)}$ for any $p \geq m(n)$. Then $\bar{E}_{n}^{E_{p}}$ is a closed subspace of the Fréchet space $\left(E_{p}, \xi_{p}\right)$, so $\left(\bar{E}_{n}^{E_{p}}, \xi_{p} \mid \bar{E}_{n}^{E_{p}}\right)$ is a Fréchet space. Since $\bar{E}_{n}^{E_{p}} \subset E_{m(n)}$ and the topology $\xi_{p} \mid E_{m(n)}$ is weaker than $\xi_{m(n)}, \bar{E}_{n}^{E_{p}}$ is a closed subspace of the Fréchet space $\left(E_{m(n)}, \xi_{m(n)}\right)$. Hence $\left(\bar{E}_{n}^{E_{p}}, \xi_{m(n)} \mid \bar{E}_{n}^{E_{p}}\right)$ is a Fréchet space too. By the open mapping theorem, $\left(\bar{E}_{n}^{E_{p}}, \xi_{m(n)} \mid \bar{E}_{n}^{E_{p}}\right)=$ $\left(\bar{E}_{n}^{E_{p}}, \xi_{p} \mid \bar{E}_{n}^{E_{p}}\right)$. Remark that $\bar{E}_{n}^{E_{m(n)}}$ is closed in $\left(\bar{E}_{n}^{E_{p}}, \xi_{m(n)} \mid \bar{E}_{n}^{E_{p}}\right)$ and dense in $\left(\bar{E}_{n}^{E_{p}}, \xi_{p} \mid \bar{E}_{n}^{E_{p}}\right)=\left(\bar{E}_{n}^{E_{p}}, \xi_{m(n)} \mid \bar{E}_{n}^{E_{p}}\right)$, we have $\bar{E}_{n}^{E_{m(n)}}=\bar{E}_{n}^{E_{p}}$. Thus $\bar{E}_{n}^{E_{m(n)}}$ is $\xi_{p}$-closed for any $p \geq m(n)$. Let $\left(F_{n}, \eta_{n}\right)=\left(\bar{E}_{n}^{E_{m(n)}}, \xi_{m(n)} \mid \bar{E}_{n}^{E_{m(n)}}\right)$, then $\left(F_{n}, \eta_{n}\right)$ is a Fréchet space and $F_{n}$ is $\xi_{p}$-closed for any $p \geq m(n)$. Particularly, $F_{n}$ is $\xi_{m(n+1)}$-closed, and $F_{n}$ is closed in $\left(\bar{E}_{n+1}^{E_{m(n+1)}}, \xi_{m(n+1)} \mid \bar{E}_{n+1}^{E_{m(n+1)}}\right)=\left(F_{n+1}, \eta_{n+1}\right)$. Let $(F, \eta)=$ ind $\lim \left(F_{n}, \eta_{n}\right)$. Then all $\left(F_{n}, \eta_{n}\right)$ are Fréchet spaces and each $F_{n}$ is closed in $\left(F_{n+1}, \eta_{n+1}\right)$. It follows from the Dieudonné-Schwartz Theorem that $(\mathrm{DST})$ holds for $(F, \eta)=$ ind $\lim \left(F_{n}, \eta_{n}\right)$. Evidently, $(E, \xi)=(F, \eta)$; [see 6, Chapter V, Supplement (3)]. Thus a set $B \subset E$ is $\xi$-bounded if and only if it is $\eta$-bounded if and only if it is contained and bounded in some $\left(F_{n}, \eta_{n}\right)$, namely, $B$ is contained and bounded in some $\left(E_{m}, \xi_{m}\right)$. Hence (DST) holds for $(E, \xi)=$ ind $\lim \left(E_{n}, \xi_{n}\right)$.

Corollary 1. Let all $\left(E_{n}, \xi_{n}\right)$ be Fréchet spaces. Then (DST) holds provided that for any $n \in N$ there is $m(n) \in N$ such that for each $p \geq m(n)$, there is a neighborhood $U_{p}$ of 0 in $\left(E_{p}, \xi_{p}\right)$ satisfying $\left(\overline{U_{p} \cap E_{n}}\right)^{E_{p}} \subset E_{m(n)}$.

Proof. Without loss of generality, we may assume that $U_{p}$ is an open absolutely convex neighborhood of 0 in $\left(E_{p}, \xi_{p}\right)$. Let $x \in U_{p} \cap \bar{E}_{n}^{E_{p}}$, then there is a sequence $\left\{x_{i}\right\} \subset E_{n}$ such that $x_{i} \stackrel{i}{\rightarrow} x$ in $\left(E_{p}, \xi_{p}\right)$. Since $U_{p}$ is $\xi_{p}$-open and $x \in U_{p}$, there is an index $i_{0}$ such that $x_{i} \in U_{p}$ for any $i \geq i_{0}$. Thus $x_{i} \in U_{p} \cap E_{n}$ for any $i \geq i_{0}$ and $x_{i} \stackrel{i}{\rightarrow} x$ in $\left(E_{p}, \xi_{p}\right)$, so $x \in\left(\overline{U_{p} \cap E_{n}}\right) E_{p}$. Namely $U_{p} \cap \bar{E}_{n}^{E_{p}} \subset\left(\overline{U_{p} \cap E_{n}}\right)^{E_{p}}$. By the hypotheses, $\left(\overline{U_{p} \cap E_{n}}\right)^{E_{p}} \subset E_{m(n)}$, so $U_{p} \cap \bar{E}_{n}^{E_{p}} \subset E_{m(n)}$. Thus $\bar{E}_{n}^{E_{p}}=E_{p} \cap \bar{E}_{n}^{E_{p}}=\left(\bigcup_{k=1}^{\infty} k U_{p}\right) \cap \bar{E}_{n}^{E_{p}}=\bigcup_{k=1}^{\infty} k\left(U_{p} \cap\right.$ $\left.\bar{E}_{n}^{E_{p}}\right) \subset E_{m(n)}$. By Theorem 1, (DST) holds.

Corollary 2. Let all $\left(E_{n}, \xi_{n}\right)$ be Fréchet spaces. Then (DST) holds provided that for any $n \in N$ there is $m(n) \in N$ such that $\bar{E}_{n}^{E} \subset E_{m(n)}$, where ${ }^{E}{ }_{n}^{E}$ is the quasi-closure [7, p. 296] of $E_{n}$ in $(E, \xi)$.

Counterexample. Put $W(x)=\sqrt{1+x^{2}}, x \in(-\infty,+\infty)$, and $E_{n}=\{f \in$ $\left.L^{2}(R):\|f\|_{n}^{2}=\int_{R}\left|W^{-n} f\right|^{2} d x<+\infty\right)$. The norm $\|\cdot\|_{n}$ makes $E_{n}$ into a Hilbert space. Let $\mathscr{D}[-n, n]=\left\{f \in C^{\infty}(R)\right.$ : $\left.\operatorname{supp} f \subset[-n, n]\right\}$ and $\mathscr{D}=$ $\bigcup_{n=1}^{\infty} \mathscr{D}[-n, n]$, then $\mathscr{D}$ is dense in each $E_{n}$, so we have $E_{n+p}=\overline{\mathscr{D}}^{E_{n+p}} \subset \bar{E}_{n}^{E_{n+p}}$ 
and the hypothesis in Theorem 1 does not hold. But, by Theorem 4 in [3], (DST) holds.

The counterexample shows that the hypothesis in Theorem 1 is not a necessary condition for (DST). However, for strict inductive limits of metrizable spaces, we have the following theorem.

Theorem 2. Let $(E, \xi)=\operatorname{ind} \lim \left(E_{n}, \xi_{n}\right)$ be a strict inductive limit of metrizable spaces. Then (DST) holds if and only if for any $n \in N$ there is $m(n) \in N$ such that $\bar{E}_{n}^{E_{p}} \subset E_{m(n)}$ for all $p \geq m(n)$.

Proof. By Theorem 2 in [5], if (DST) holds then for any $n \in N$ there is $m(n) \in$ $N$ such that $\bar{E}_{n}^{E} \subset E_{m(n)}$. Since $\bar{E}_{n}^{E_{p}} \subset \bar{E}_{n}^{E}$ for all $p \geq m(n)$, the necessity is proved.

Conversely, assume that the condition is satisfied and there is a set $B$ bounded in $(E, \xi)$ which is not contained in any $E_{n}$. Choose a sequence $1=n_{1} \leq m\left(n_{1}\right)<n_{2} \leq m\left(n_{2}\right)<n_{3} \leq m\left(n_{3}\right)<n_{4} \leq \cdots$ such that $\bar{E}_{n_{k}}^{E_{p}} \subset$ $E_{m\left(n_{k}\right)} \subset E_{n_{k+1}}$ and $b_{k} \in B \cap\left(E_{n_{k+1}} \backslash \bar{E}_{n_{k}}^{E_{p}}\right)$ for all $p \geq m\left(n_{k}\right), k \in N$. Since $(1 / k) b_{k} \in E_{n_{k+1}} \backslash \bar{E}_{n_{k}}^{E_{n_{k+1}}}$ and $\left(E_{n_{k}}, \xi_{n_{k}}\right)$ is a topological subspace of $\left(E_{n_{k+1}}, \xi_{n_{k+1}}\right)$, there is an absolutely convex neighborhood $U_{n_{k}}$ of 0 in $\left(E_{n_{k}}, \xi_{n_{k}}\right)$ such that $U_{n_{k+1}} \cap E_{n_{k}}=U_{n_{k}}$ and $(1 / k) b_{k} \notin U_{n_{k+1}}$; see [8, Lemma 13-3-2]. Put $U=\bigcup_{k=1}^{\infty} U_{n_{k}}$, then $U$ is a neighborhood of 0 in $(E, \xi)$ which does not contain any $(1 / k) b_{k}$, a contradiction. Thus $B$ is contained in some $E_{n}$ and (DS) holds. Since $\xi \mid E_{n}=\left(E_{n}, \xi_{n}\right)$ for any $n \in N$, we have (DST) holds.

2. Next we shall give some interesting results for bounded sets in inductive limits of Fréchet spaces.

An absolutely convex subset $B$ of a locally convex space is called a barrelled disk (respectively, Banach disk) if $\operatorname{sp}[B]$ with the gauge of $B$ is a barrelled space (respectively, Banach space). Obviously each Banach disk is a barrelled disk. But the converse is not true.

Theorem 3. Let all $\left(E_{n}, \xi_{n}\right)$ be Fréchet spaces and a set $B$ contained and closed in some $\left(E_{n}, \xi_{n}\right)$. If $B$ is a barrelled disk (or Banach disk) then $B$ is bounded in $\left(E_{n}, \xi_{n}\right)$.

Proof. Let $U_{n}^{(1)} \supset U_{n}^{(2)} \supset U_{n}^{(3)} \supset \cdots$ be a base of closed absolutely convex neighborhoods of 0 in $\left(E_{n}, \xi_{n}\right)$. Put $E_{B}=\mathrm{sp}[B]$, then $E_{B} \subset E_{n}$. The collection of all sets $\left(B / 2^{k-1}\right) \cap U_{n}^{(k)}, k=1,2,3, \cdots$, forms a base of neighborhoods of 0 for some locally convex topology on $E_{B}$. We denote the topology on $E_{B}$ by $\eta$. Obviously $\left(E_{B}, \eta\right)$ is metrizable. Let $\left\{x_{i}\right\}$ be a Cauchy sequence in $\left(E_{B}, \eta\right)$, then $\left\{x_{i}\right\}$ is also a Cauchy sequence in $\left(E_{n}, \xi_{n}\right)$. Since $\left(E_{n}, \xi_{n}\right)$ is complete, there is $x_{0} \in E_{n}$ such that $x_{i} \stackrel{i}{\rightarrow} x_{0}$ in $\left(E_{n}, \xi_{n}\right)$. For any fixed $k \in N$, there is $i_{k} \in N$ such that $x_{i}-x_{j} \in\left(B / 2^{k-1}\right) \cap U_{n}^{(k)}$ for $i, j \geq i_{k}$. 
Since $\left(B / 2^{k-1} \cap U_{n}^{(k)}\right.$ is closed in $\left(E_{n}, \xi_{n}\right)$ and $x_{j} \stackrel{j}{\longrightarrow} x_{0}$ in $\left(E_{n}, \xi_{n}\right)$, we have $x_{i}-x_{0} \in\left(B / 2^{k-1}\right) \cap U_{n}^{(k)}$ for $i \geq i_{k}$. Thus $x_{i} \stackrel{i}{\rightarrow} x_{0}$ in $\left(E_{B}, \eta\right)$. Hence $\left(E_{B}, \eta\right)$ is a Fréchet space. Suppose that $p_{B}$ is the gauge of $B$, then $\left(E_{B}, p_{B}\right)$ is a barrelled space. Evidently the identity mapping id: $\left(E_{B}, \eta\right) \rightarrow\left(E_{B}, p_{B}\right)$ is continuous. By the open mapping theorem, id is open. Hence for each $k \in N$, there is $\lambda_{k}>0$ such that $\left.\left.\lambda_{k} B \subset\left(B / 2^{k-1}\right) \cap U_{n}^{(k)}\right) \subset U_{n}^{(k)}\right)$. Namely $B$ is absorbed by each $U_{n}^{(k)}$, so $B$ is bounded in $\left(E_{n}, \xi_{n}\right)$. This completes the proof.

Let $(E, \xi)=$ ind $\lim \left(E_{n}, \xi_{n}\right)$ be an inductive limit of locally convex spaces and a set $B$ contained in some $E_{n}$. If $B$ is $\xi_{n}$-bounded then $B$ is $\xi_{m}$-bounded for any $m>n$. However $B \quad \xi_{m}$-bounded (for some $m>n$ ) does not imply $B \xi_{n}$-bounded because the topology $\xi_{n}$ is stronger than $\xi_{m} \mid E_{n}$.

Using Theorem 3, we obtain the following two elegant results.

Theorem 4. Let all $\left(E_{n}, \xi_{n}\right)$ be Fréchet spaces and an absolutely convex set $B$ contained in $E_{n}$. If there is $m>n$ such that $B$ is bounded and closed in $\left(E_{m}, \xi_{m}\right)$, then $B$ is bounded and closed in $\left(E_{n}, \xi_{n}\right)$.

Proof. Since $B$ is closed in $\left(E_{m}, \xi_{m}\right)$ and the topology $\xi_{n}$ is stronger than $\xi_{m} \mid E_{n}, B$ is closed in $\left(E_{n}, \xi_{n}\right)$. By the hypothesis, there is $m>n$ such that $B$ is bounded and closed in the Fréchet space $\left(E_{m}, \xi_{m}\right)$, so $B$ is a Banach disk. By Theorem 3,B is bounded in $\left(E_{n}, \xi_{n}\right)$.

Theorem 5. Let all $\left(E_{n}, \xi_{n}\right)$ be Fréchet spaces and (DST) hold. If an absolutely convex set $B \subset E_{n}$ is bounded and closed in $(E, \xi)$, then $B$ is bounded and closed in $\left(E_{n}, \xi_{n}\right)$.

Proof. Since (DST) holds, $B$ is bounded in $\left(E_{m}, \xi_{m}\right)$ for some $m \geq n$. By the hypotheses, $B$ is $\xi$-closed, hence also $\xi_{m}$-closed. By Theorem $4, B$ is bounded and closed in $\left(E_{n}, \xi_{n}\right)$.

\section{ACKNOWLEDGEMENT}

The author wishes to thank the referee for his helpful suggestions.

\section{REFERENCES}

1. J. Horváth, Topological vector spaces and distributions, Vol. 1, Addison-Wesley, Reading, Massachusetts, 1966.

2. J. Kucera and K. McKennon, Bounded sets in inductive limits, Proc. Amer. Math. Soc. 69 (1978), 62-64.

3. __ Dieudonné-Schwartz theorem on bounded sets in inductive limits, Proc. Amer. Math. Soc. 78 (1980), 366-368.

4. J. Kucera and C. Bosch, Dieudonné-Schwartz theorem on bounded sets in inductive limits II, Proc. Amer. Math. Soc. 86 (1982), 392-394.

5. Qiu Jing Hui, Dieudonné-Schwartz theorem in inductive limits of metrizable spaces, Proc. Amer. Math. Soc. 92 (1984), 255-257.

6. A. P. Robertson and W. J. Robertson, Topological vector spaces, Cambridge University Press, London, 1964. 
7. G. Köthe, Topological vector spaces I, Springer-Verlag, Berlin, Heidelberg, New York, 1983.

8. A. Wilansky, Modern methods in topological vector spaces, Blaisdell, 1978.

Department of Mathematics, Suzhou University, Suzhou, Jiangsu, People's Republic OF CHINA

Current address: Beijing University of Iron \& Steel Technology, Room 404, Building No. 10, Beijing, People's Republic of China 